\title{
INFLUENCE OF BRASSICA SPROUTS ON SHORT CHAIN FATTY ACIDS CONCENTRATION IN STOOLS OF RATS WITH THYROID DYSFUNCTION
}

\author{
JUSTYNA DOBROWOLSKA-IWANEK*, PAWEE ZAGRODZKI, \\ EWELINA PROCHOWNIK, ANETA JARKIEWICZ and PAWEŁ PAŚKO
}

\author{
Department of Food Chemistry and Nutrition, \\ Jagiellonian University Medical College, Kraków, Poland
}

\begin{abstract}
The aim of the study was to investigate the influence of kohlrabi and broccoli sprouts added to the diet on the concentrations of short-chain fatty acids in feces samples of rats with thyroid dysfunction. Two models of hypothyroidism were used. The first one was based on the diet with iodine deficiency and the second by sulfadimethoxine addition to the animal drinking water. In most cases, the concentrations of acetic acid, propanoic acid and butanoic acid in stool samples obtained from rats with thyroid dysfunction were insignificantly lower than found in the control group. Moreover, almost in all cases the diet enriched with brassica sprouts insignificantly increased the concentration of fatty acids in investigated material. A significant positive correlation between concentrations of butanoic and acetic acids was found in different groups of rats. In conclusion, the addition of broccoli or kohlrabi sprouts in rat's diet may slightly alleviate the decline of concentrations of some fatty acids in the stool of animals with thyroid dysfunction. Long term effects of this phenomenon should be evaluated in future studies.
\end{abstract}

Keywords: broccoli sprouts; thyroid dysfunction; brassica vegetables; short-chain fatty acids; stool samples

Different thyroid diseases have become more common in recent years. They have an influence on various body systems, among others also on the function of the gastrointestinal tract. Hypothyroidism may be associated with an oesophageal motility disorder presenting as dysphagia or heartburn with subsequent symptoms like dyspepsia, nausea, or vomiting caused by delayed gastric emptying. Abdominal discomfort, flatulence, and bloating may also occur in patients with hypothyroidism and bacterial overgrowth in their digestive system (1). Thus the effect of hypothyroidism on the gastrointestinal tract seems to be multifactorial with possible further alterations in hormone side effects, as well as neuromuscular disorders and myopathy caused probably by mucopolysaccharide accumulation in gastrointestinal tissue $(2,3)$. Maser et al. indicated that the effect of thyroid hormones on the gastrointestinal tract may be either direct or mediated through the adrenergic/catecholamine system (4). Hypothyroid3ism causes hypomotility. Gullo et al. (5) indicated that in hypothyroid patients pancreatic secretion of bicarbonate and enzymes decreased significantly, and concluded that the thyroid gland plays an essential role in maintaining the functional integrity of the exocrine pancreas. However, there is no direct relation between the changes in the kinetic of the gastrointestinal tract and the level of hypothyroidism $(3,6)$.

Hypothyroidism intrinsically results in the accumulation of hyaluronic acid in the gastric smooth muscle, in the skin, heart muscle, and skeletal muscles. This leads to interstitial edema. Edema in the gastric muscle may predispose to abnormalities of gastric myoelectric activity (4).

Apart from causing delays of gastric emptying, the hypothyroid state is also associated with a decrease in duodenal basal electrical rhythm. Reduction of peristalsis in hypothyroidism is the main pathophysiologic process, and constipation remains the most frequent gastrointestinal complaint (2). Constipation may result from diminished motility, leading to an ileus, megacolon, or rarely pseudoobstruction with fecal impaction and megacolon (7). Tenore et al. (8) indicated that thyroid hormone deficiency may influence transepithelial flux transport by inhibiting $\mathrm{Cl}^{-} / \mathrm{HCO}_{3}$ - anion exchange with a sub-

\footnotetext{
* Corresponding author: e-mail: justyna.dobrowolska-iwanek@uj.edu.pl
} 
sequent effect on intestinal motility. Hypothyroidism can be also associated with insulin resistance, changes in lipid metabolism and instead should be increased risk of obesity $(9,10)$.

Short-chain fatty acids (SCFAs), primarily acetic acid, propanoic acid and butanoic acid, are the major end products generated by the anaerobic microbiota in large bowel as the result of the bacteria fermentation process of indigestible carbohydrates (11). These acids can prevent the overgrowth of $\mathrm{pH}$-sensitive pathogenic microorganisms by lowering the $\mathrm{pH}$ of the luminal environment (12). It is well known that oxidation of SCFAs and especially of butanoic acid supplies the intestinal epithelial cells with $60-70 \%$ of energy requirements for their proliferation and differentiation (13). In addition, butyrate participates in the regulation of mucin protein production which results in changes in bacterial adherence in the gut (14). Butyrate by inhibition of histone deacetylase and the suppression of NF- $\kappa \mathrm{B}$ activity reduces the expressions of cytokine genes for TNF $\alpha$, IL1 $\beta$, IL2, IL6, IL8, and IL12, and thus may affect the inflammation, colonic carcinogenesis and oxidative stress in intestines (15). SCFAs may reduce the amount of fatty tissue and, additionally, decrease body weight by increasing the synthesis of YY peptide which is responsible for satiety (16). Frost et al. indicated that acetic acid may decrease appetite through such actions like the impact on hypothalamus neurons, activation of acetyl-CoA carboxylase as well as changes in the expression of regulatory neuropeptides (17). Tolhurst et al. postulated the link between bacterial fermentation of prebiotics to SCFAs and the modulation of the production of gut hormones controlling insulin release and appetite (18). These acids cause also an increase in glucagonlike peptide-1 (GLP-1) level a decrease in insulin resistance (18) observed in hypothyroidism (10). It is worth to note, that short fatty acids evaluated in the present study may play a role in the regulation of lipid metabolism via lipolysis inhibition (19-21).

There is the link between triiodothyronine (T3), which induces intestinal alkaline phosphatase and represses lactase gene transcription in enterocytes (regulating in this way their differentiation and function), and SCFAs, whose production by the resident microbiota in the intestine lumen is enhanced in such conditions. Eventually, both T3 and SCFAs cooperate in maintaining the proper intestinal epithelial development and homeostasis (22).

Because of such combined effects of SCFAs, we have decided to evaluate the influence of thyroid imbalance on the level of SCFAs in stools of male rats and additionally to investigate the effect of two different kinds of brassica sprouts added to their diet. For the experiment, we have chosen the sprouts which have proven beneficial influence on the living organisms: broccoli sprouts and the example of new functional food - kohlrabi sprouts. During the experiment, two independent models of hypothyroidism were introduced. The first model was based on a diet with iodine deficiency causing thyroid hyperplasia, and the second on sulfadimethoxine (SDM) added to the animal drinking water as an ingredient $(0.025 \%)$ causing thyroid damage by inhibiting thyroid hormone synthesis $(23,24)$. A detailed description of the experiment and observed changes in TSH and thyroid hormones was published recently by Paśko et al. (25).

\section{METHODS AND MATERIALS}

\section{Plant material}

Voucher specimens of broccoli seeds (Brassica oleracea convar. botrytis var. cymosa) and kohlrabi seeds (Brassica oleracea convar. acephala var. gangylodes) were deposited in the Department of Food Chemistry and Nutrition, Faculty of Pharmacy, Jagiellonian University Medical College (No\#BOCB/PP/PL 1036 and No\#BOAG/PP/PL 1043 respectively). Four days old sprouts were harvested by the Uniflora Company, Poland. After sprouting, these materials were lyophilized to obtain dry material suitable for the preparation of animal fodder. There was not a significant difference in the total amount of water in both investigated sprouts. Brassica sprouts were rich source of phenolic compounds such as flavonoids, phenolic acids and unsaturated fatty acids. Broccoli sprouts were a significant source of sufhoraphane in comparison to kohlrabi sprouts (26).

\section{Animals}

The 108 male (mean weight $256 \pm 19$ g) 4week-old Wistar rats were maintained in plastic cages in an air-conditioned animal room in the Animal House of the Faculty of Pharmacy, Jagiellonian University Medical College for one week before the experiment started (temperature 22 $\pm 2^{\circ} \mathrm{C}$, with a relative humidity of $50 \pm 5 \%, 12 \mathrm{~h}$ periods of light and darkness). After 1 week of acclimatization, rats were divided into 9 groups, each consisting of 12 animals and fed one of the following diets: standard diet (C); iodine deficiency diet (DI); diet with $7 \%$ of lyophilized broccoli sprouts (B); diet with $7 \%$ of lyophilized kohlrabi sprouts $(\mathrm{KH})$; iodine deficiency diet with $7 \%$ of lyophilized broccoli sprouts (BDI); iodine deficien- 
cy diet with $7 \%$ of lyophilized kohlrabi sprouts (KHDI); standard diet with $0.025 \%$ sulfadimethoxine (SDM) administered in animal drinking water (S); diet with $7 \%$ of lyophilized broccoli sprouts and with $0.025 \%$ SDM administered in their drinking water (BS) or a diet with $7 \%$ of lyophilized kohlrabi sprouts and with $0.025 \%$ SDM administered in their drinking water (KHS). The rats had unlimited access to fodder and water. Diets were prepared by The Morawski Fodder Company (Poland). All compounds present in all variants of the diets (apart from $\mathrm{C}, \mathrm{B}$ and $\mathrm{KH}$ group) were certified as being without iodine. Detailed descriptions of the composition of the diets and intake of the fodder are available in Table 1. The protocols for animal experiments were approved by the Animal Experimentation Committee of Jagiellonian University, Kraków, Poland. After 8 weeks, at the end of the experiment, the stool was collected and samples were stored at $-30^{\circ} \mathrm{C}$.

\section{Chemicals}

Deionized water of $18 \mathrm{M} \Omega \mathrm{cm}$ was obtained from Milli Ro \& Q water purification system (Merck-Millipore, Billerica, MA, USA). Sulfadimethoxine, acetonitrile (ACN), 70\% perchloric acid, 99\% acetic acid, 99.5\% propanoic acid, 99.5\% butanoic acid were produced by Sigma-Aldrich (Steinheim, Germany), 98\% diethylamine and 85\% orthophosphoric acid were purchased from Merck (Germany).
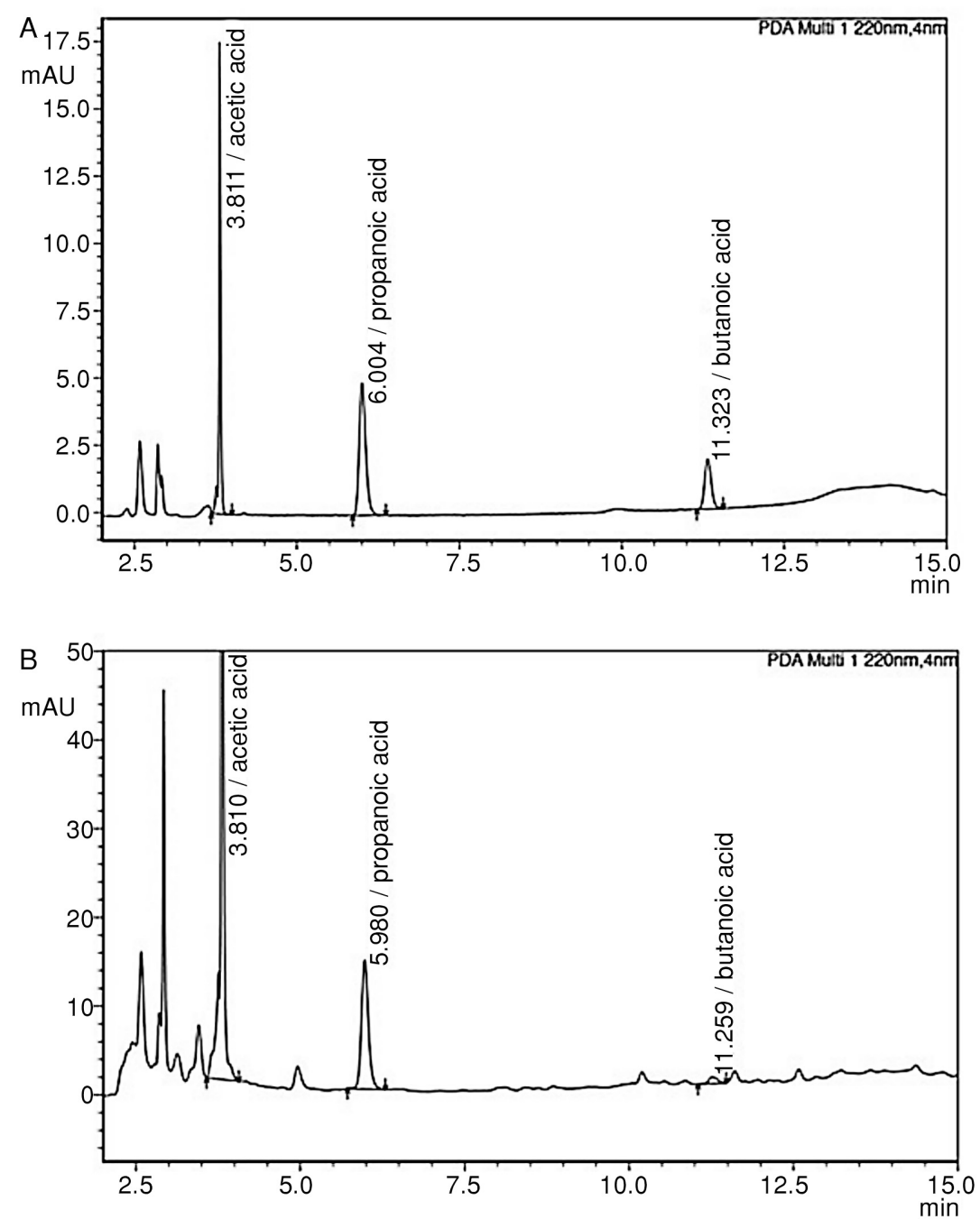

Figure 1. Chromatograms of HPLC analysis of a standard mixture of three short-chain organic acids (chromatogram A) and an examplary sample of rat stool extract (chromatogram B). 


\section{Feces samples preparation}

Stool samples (0.8-1.9 g wet sample) were dried in a forced air-drying oven at $40{ }^{\circ} \mathrm{C}$ for $48 \mathrm{~h}$, weighted again and transferred into $15 \mathrm{~mL}$ plastic vials. The extraction procedure was carried out according to the method described in a previous report by Dobrowolska-Iwanek et al. (27). First, dry feces samples (about $0.3 \mathrm{~g}$ ) were immersed in $5 \mathrm{~mL}$ of $0.15 \mathrm{~mol} / \mathrm{L}$ perchloric acid and exposed to ultrasounds (at $35^{\circ} \mathrm{C}$ ) for $40 \mathrm{~min}$. In the next step, the contents of vessels were shaken for 4 min and supernatants were decanted into $15 \mathrm{~mL}$ tubes. Remaining stools pellets were subjected to extraction procedure two more times and every time the supernatant was transferred into $15 \mathrm{~mL}$ tube. Finally, the contents of each vial were made up to $14 \mathrm{~mL}$ with perchloric acid solution and centrifuged for $10 \mathrm{~min}$ at 10000 $\mathrm{rpm}$ at $4^{\circ} \mathrm{C}$. The extracts were stored at $-20^{\circ} \mathrm{C}$ until analysis. Before measurement, samples were appropriately diluted in $0.15 \mathrm{~mol} / \mathrm{L}$ perchloric acid and filtered through a nylon 0.22-micrometer syringe filter of a diameter of $13 \mathrm{~mm}$ (Labe Ltd. Filter-Bio, China). Organic acids in stool samples were determined by a high-performance liquid chromatography (HPLC) method.

\section{Instrumentation}

The concentrations of organic acids were measured by using a high-performance liquid chromatography system (Prominence, Shimadzu, Japan) consisted of a Shimadzu LC-20AD liquid chromatography SIL-20A autosampler and SPDM20A-Diode Array Detector. The analytes were separated in a Synergi $4 \mathrm{u}$ Hydro-RP $80 \mathrm{~A} \mathrm{C}_{18}$ column $(250 \times 4.6 \mathrm{~mm}$ I.D. $4 \mu \mathrm{m})$ thermostated to $35^{\circ} \mathrm{C}$. The mobile phase was prepared by mixing ACN with diluted orthophosphoric acid $(\mathrm{pH} 2.6$, $1 \%$ diethylamine) in the appropriate ratio (both solutions were degassed by ultrasonication before use). The gradient program: 0 min $20 \% \mathrm{ACN}, 6$ $\min 30 \%, 12 \min 30 \%, 16 \min 40 \%, 25 \min 10 \%$ and $35 \mathrm{~min} 10 \%$ was used. The injection volume was $75 \mu \mathrm{L}$ and the flow rate was $1 \mathrm{~mL} / \mathrm{min}$. The wavelength selected for the quantitation of the organic acids was $220 \mathrm{~nm}$.

\section{Validation parameters}

The acquired signals (peaks) in the HPLC analysis were identified based on the retention time compared with those of pure compounds and spiked samples to ensure the correctness of the identification. For quantification of organic acids, the external calibration curve was calculated by the analysis of calibrators at following concentration levels: $6.25,12.5,25,50$, and $100 \mathrm{mg} / \mathrm{L}$ for acetic and propanoic acids, and 3.125, 6.25, 12.5, 25, $50 \mathrm{mg} / \mathrm{L}$ for butanoic acid. Obtained correlation coefficients of calibration curves were over 0.999 . Recovery rates of all analytes were between 99.9 and $106.1 \%$. The precision which involved repeatability of analysis $(n=6)$, was evaluated using fecal extracts. Relative standard deviations were equal or less than $2.1 \%$. Chromatograms of HPLC analysis a standard mixture of three short-chain organic acids and an example of fecal extract were demonstrated in Figure 1.

Table 1. Detailed composition of the diet.

\begin{tabular}{|c|c|c|c|c|c|c|}
\hline \multicolumn{7}{|c|}{ Fodder composition (g/kg fodder) } \\
\hline & C & DI & B/KH & BDI/KHDI & S & BS/KHS \\
\hline Gluten & 200 & 200 & 200 & 200 & 200 & 200 \\
\hline Sprouts & - & - & 70 & 70 & - & 70 \\
\hline L-cystein & 3 & 3 & 3 & 3 & 3 & 3 \\
\hline Starch & 532 & 532 & 462 & 462 & 532 & 462 \\
\hline Sucrose & 100 & 100 & 100 & 100 & 100 & 100 \\
\hline Corn oil & 70 & 70 & 70 & 70 & 70 & 70 \\
\hline Cellulose & 50 & 50 & 50 & 50 & 50 & 50 \\
\hline AIN-93G & 35 & - & 35 & - & 35 & 35 \\
\hline AIN-93G $^{\mathrm{b}}$ & - & 35 & - & 35 & - & - \\
\hline AIN-93G $^{\mathrm{c}}$ & 10 & 10 & 10 & 10 & 10 & 10 \\
\hline Total g & 1000 & 1000 & 1000 & 1000 & 1000 & 1000 \\
\hline
\end{tabular}

${ }^{a}$ standard mixture of essential and trace elements; ${ }^{b}$ standard mixture of essential and trace elements without iodine; 'standard mixture of vitamins; " sprouts - either broccoli sprouts (B, BDI, BS), or kohlrabi sprouts (KH, KHDI, KHS) were added to the diet. 


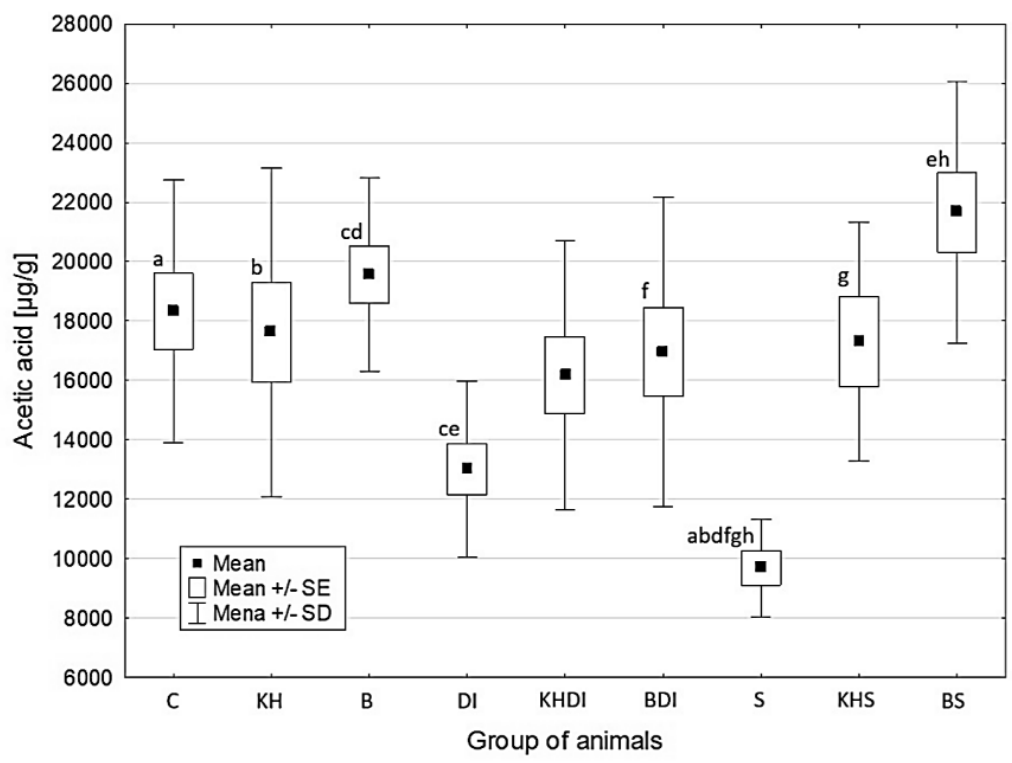

Figure 2. Mean concentrations of acetic acid in stool samples, in rat groups fed one of the following diets: standard diet (C); iodine deficiency diet (DI); diet with broccoli sprouts (B); diet with kohlrabi sprouts (KH); iodine deficiency diet with broccoli sprouts (BDI); iodine deficiency diet with kohlrabi sprouts (KHDI); standard diet SDM (S); diet with broccoli sprouts and with SDM (BS), diet with kohlrabi sprouts and SDM (KHS); SE - standard error, SD - standard deviation; the same letters indicate significant differences in mean values in respective groups $(\mathrm{p}<5.05)$.

\section{Statistical methods}

Descriptive statistics were calculated for all parameters and shown in Figures 2-4. Comparisons between groups were performed using ANOVA with Tukey post hoc test. Differences with $\mathrm{p}<0.05$ were considered as statistically significant. The associations between pairs of parameters were quantified by calculating Pearson correlation parameters. These statistical analyses were carried out using package STATISTICA v. 12 (Statsoft, Tulsa, OK, USA). Fisher's two-tailed test was used to assess the significance of the difference between the subsequent two Pearson correlation coefficients. This part of statistical evaluations was done by means of the online calculator (http://vassarstats.net/rdiff.html (28)) for Fisher R-to-z transformation.

\section{RESULTS}

The purpose of the study was to examine the impact of the thyroid imbalance on the concentration of acetic acid, propanoic acid and butanoic acid in rat stool samples. Moreover, the effects of a diet enriched with kohlrabi and broccoli sprouts on SCFAs content in feces samples received from examined groups of rats were analyzed. Study results were summarized in Figures 2-4. As a general rule, acetic acid was present in the highest con- centrations among determined organic acids in all investigated samples, followed by propanoic acids and butanoic acid.

There were no significant differences between mean concentrations of acetic acid, propanoic acid and butanoic acid in feces of rats fed a diet with broccoli and kohlrabi sprouts (B and $\mathrm{KH}$ ) and control group (C). Contents of all organic acids in stool samples obtained from rats with thyroid imbalance, triggered by iodine deficiency diet (DI) or sulfadimethoxine addition to the drinking water (S), were lower than found in control group (there was no significant difference between results obtained for rats from S and DI group). However, only in the case of acetic acid significant difference was found between results obtained for the control group and $\mathrm{S}$ group $(\mathrm{p}$ $<0.05$ ). In stool samples from animals with hyperplasia (DI) the average concentration of butanoic acid and acetic acid was slightly lower compared to those obtained from rats with the same disease but fed diets with sprouts (BDI and KHDI). Moreover, a diet with kohlrabi sprouts slightly decreased, while a diet with broccoli sprouts slightly increased concentrations of propanoic acid in rats fed iodine deficiency diet $(p>0.05)$. Results showed that diet enriched with brassica sprouts proved to be the most effective in the group of rats with thyroid damage caused by SMD. Higher organic acids concentra- 


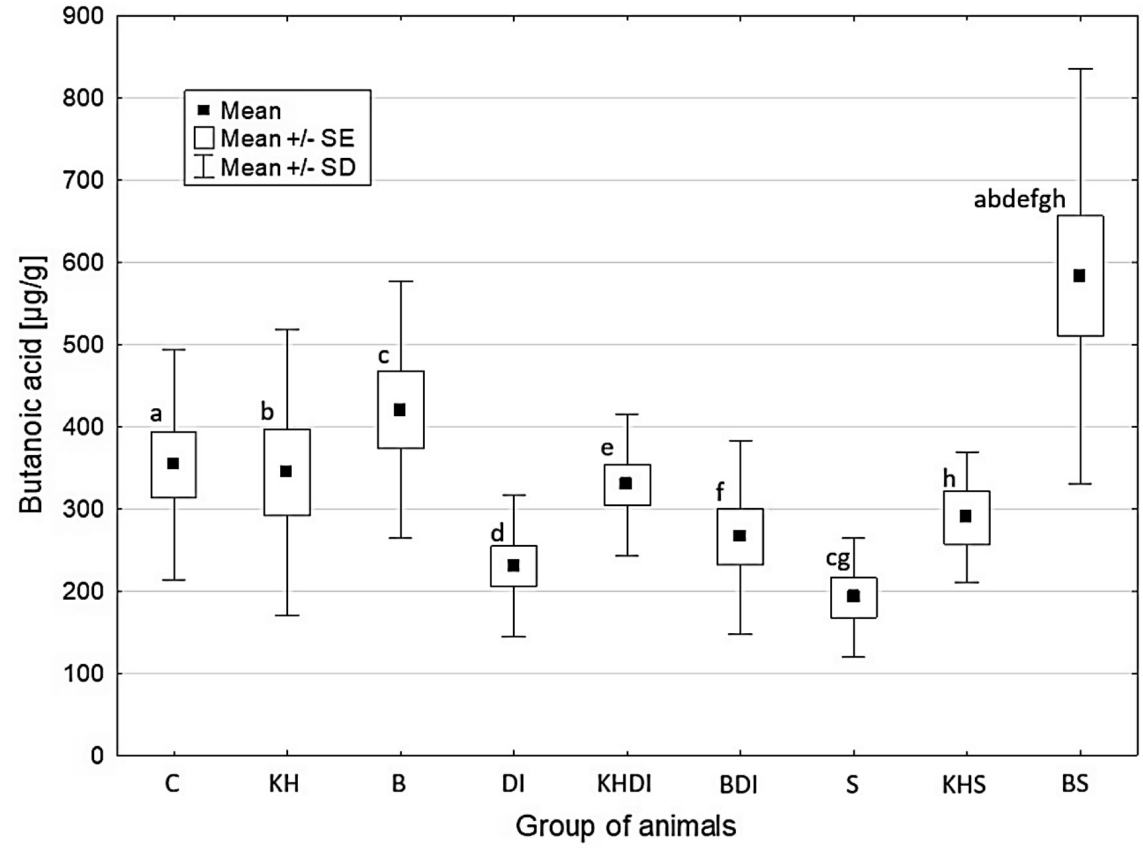

Figure 3. Mean concentrations of butanoic acid in stool samples, in rat groups fed one of the following diets: standard diet (C); iodine deficiency diet (DI); diet with broccoli sprouts (B); diet with kohlrabi sprouts (KH); iodine deficiency diet with broccoli sprouts (BDI); iodine deficiency diet with kohlrabi sprouts (KHDI); standard diet SDM (S); diet with broccoli sprouts and with SDM (BS), diet with kohlrabi sprouts and SDM (KHS); SE - standard error, SD - standard deviation; the same letters indicate significant differences in mean values in respective groups $(\mathrm{p}<0.05)$

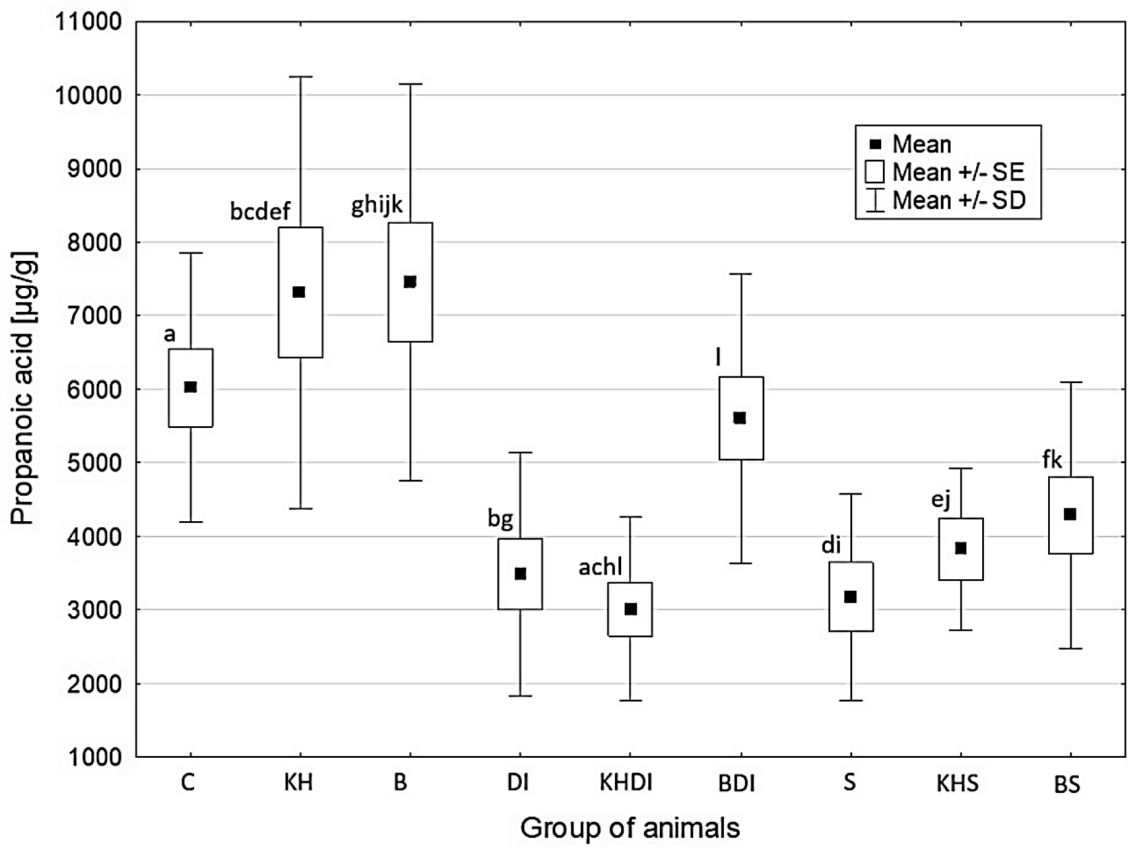

Figure 4. Mean concentrations of propanoic acid in stool samples, in rat groups fed one of the following diets: standard diet (C); iodine deficiency diet (DI); diet with broccoli sprouts (B); diet with kohlrabi sprouts (KH); iodine deficiency diet with broccoli sprouts (BDI); iodine deficiency diet with kohlrabi sprouts (KHDI); standard diet SDM (S); diet with broccoli sprouts and with SDM (BS), diet with kohlrabi sprouts and SDM (KHS); SE - standard error, SD - standard deviation; the same letters indicate significant differences in mean values in respective groups $(\mathrm{p}<0.05)$. 
tions were found in fecal samples of rats fed with sprouts (i.e. KHS and BS) than in samples from group fed with basic diet, but the differences were significant only for acetic acid.

In addition, correlations between the concentration of determined acids in feces samples of rats divided into various subgroups were analyzed. Calculations were performed for following groups: (1) - healthy rats $(\mathrm{C}, \mathrm{B}, \mathrm{KH}),(2)$ - rats with thyroid imbalance (DI, BDI, KHDI, S, BS, KHS), (3) - rats fed diets with sprouts (B, KH, BDI, KHDI, BS, KHBS), (4) - rats fed diet without sprouts (C, DI, $\mathrm{S})$. In each group positive correlation $(p<0.05)$ between concentrations of butanoic and acetic acid was found, and an example of such correlation was shown in Figure 5. There were no significant differences between corresponding correlation coefficients in various groups. Moreover, in group (4) correlation between concentrations of acetic acid and propanoic acid was revealed ( $<$ < 0.05) (Fig. 6).

\section{DISCUSSION}

Both thyroid damage models (dietary iodine deficiency and sulfadimethoxine ingestion) caused a decrease in the level of all evaluated acids compared to the control group, but there were no significant differences between these models. Such a tendency could reflect the changes in food passage in the gut which can be modified by thyroid hormones. These hormones may influence gut motility modulating neurological and smooth muscle function. In hypothyroidism state, it may be associated with decreased frequency of rhythmic colonic activity and slower oro-cecal transit time both in animals and humans (29). Slower intestinal transit of fodder may decrease the amount of carbohydrates which are substrates for SCFAs synthesis in large intestine. In rat fodder, starch, sucrose, cellulose, and sprouts are the source of carbohydrates. This is in agreement with results reported by El Oufir et al. (30) who showed that transit time is related to the nature and amount of substrates delivered to the colon, and thus may be an important factor accounting for interindividual differences in SCFAs composition.

The second factor which can have an influence on the SCFAs is the colon microbial environment. According to Flint et al. (31) bacteria responsible for the utilization of dietary polysaccharides in the gut belong to two phyla: Bacteroidetes and Firmicutes. The Gram-negative Bacteroidetes phylum includes the genera Bacteroidetes, Prevotella, Parabacteroides, and Alistipes. In contrast, the role of the intestinal indigenous microorganisms in a hypothyroid state like Hashimoto disease and dysbiosis during thyroid hormone disturbances has received little attention (32). It was suggested by Di Stefano et al. (33) that dysbiosis could affect thyroid hormone synthesis and metabolism, and gut bacteria can deiodinase thyroid hormones, thus affecting serum lev-

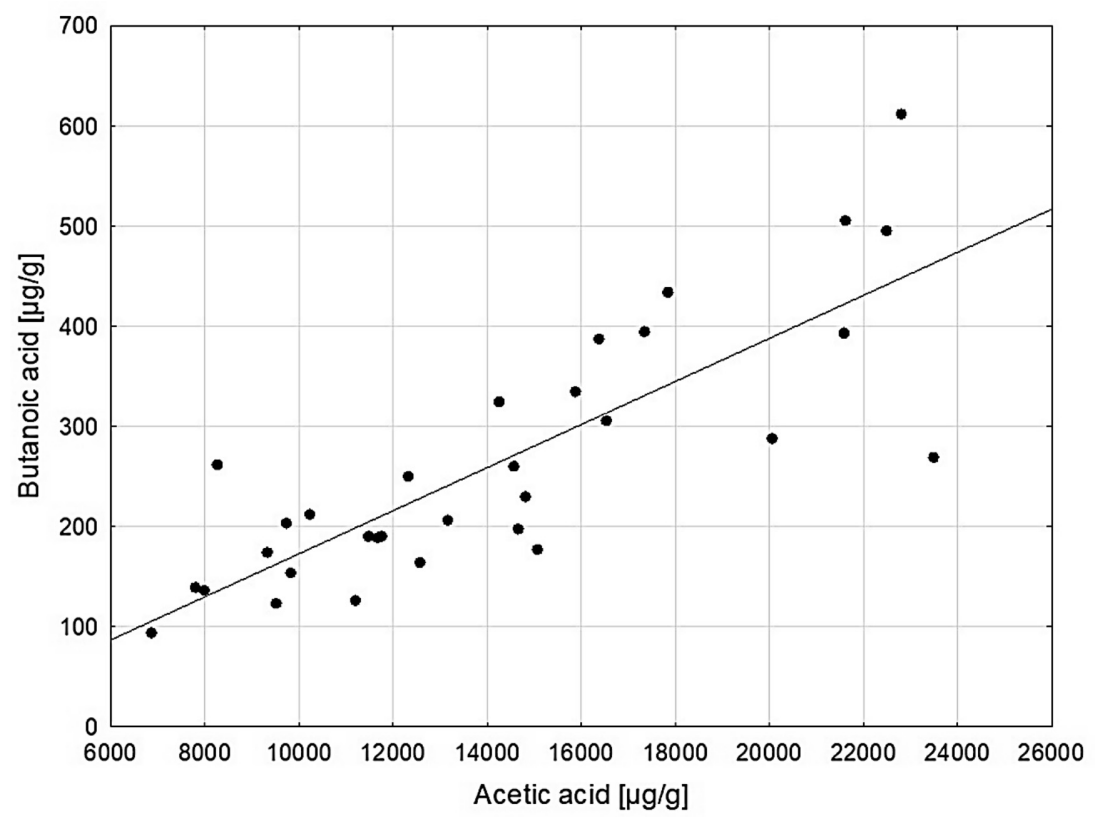

Figure 5. Correlation between concentrations of butanoic acid and acetic acid in stool samples of rats fed diet without sprouts $(\mathrm{R} 2=0.673$, $\mathrm{p}<0.05)$. 


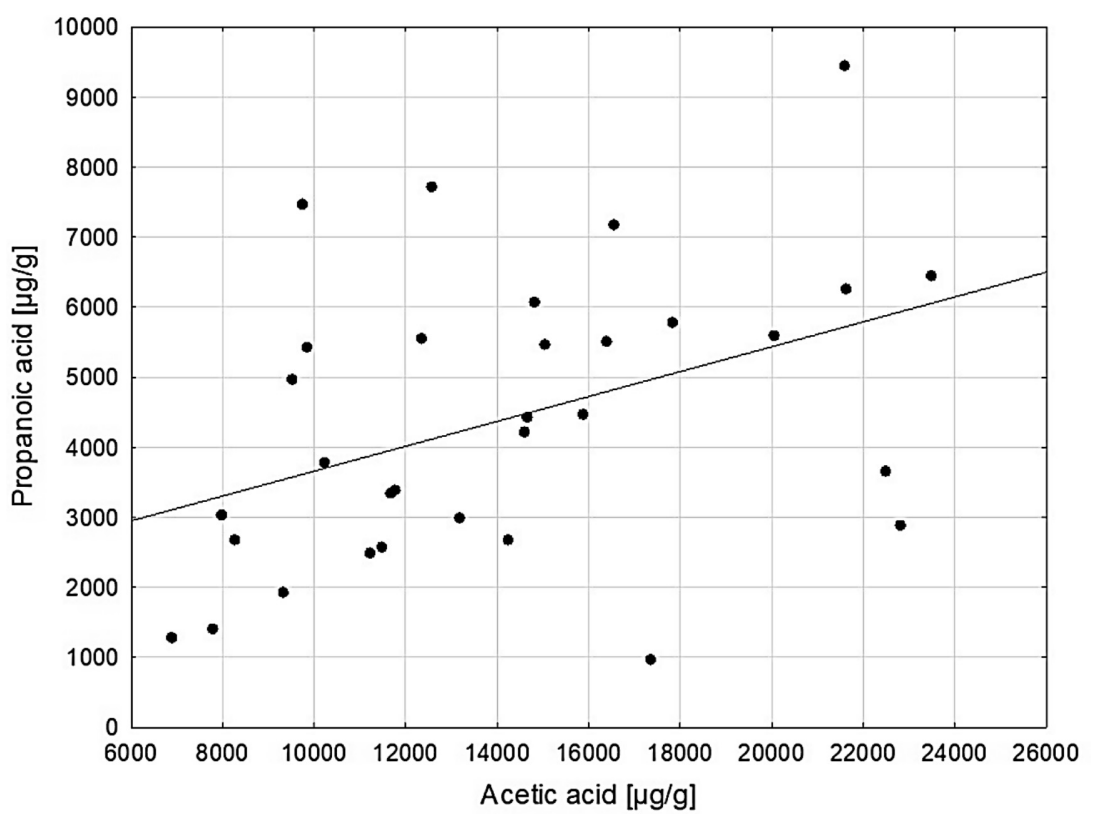

Figure 6. Correlation between concentrations of propanoic acid and acetic acid in stool samples of rats fed diet without sprouts $\left(\mathrm{R}^{2}=0.172\right.$, $\mathrm{p}<0.05)$.

els of these hormones (34). Such interactions need further study to be clarified.

The influence of polyphenolic compounds on the SCFAs level is not clear. Proanthocyanidins in apples inhibited the human microbiota (in vitro) and also suppressed SCFAs formation from carbohydrates (35). On the other hand, Boto-Ordonez et al. (36) indicated that the patients with the highest fecal Bifidobacteria concentration had a relatively high urinary concentration of syringic acid, p-coumaric acid, 4-hydroxybenzoic acid, and anthocyanin metabolites, which suggests that polyphenols apparently did not hamper in vivo gut microbiota.

Kaczmarek et al. (37) investigated the influence of material similar to that used in our experiment - broccoli florets (as a possibly good source of polyphenols and sulfur compounds) on gastrointestinal microbiota. In fact, bacterial communities were impacted by dietary intervention. The significant change in the proportion of Bacteroidetes to Firmicutes was observed, as participants consuming broccoli had increased Bacteroides by $6 \%$. Generally, this observation seems to be in agreement with our results where broccoli sprouts caused an increased concentration of SCFAs in stool in comparison to kohlrabi sprouts which are not good source of sulforaphane. In case of butanoic acid concentration, the highest level was noted in BS group and the reason for that is not clear because it is known that the Firmicutes have been identified as the dominant producers of butyrate in the colon (31) and they should be decreased in our experiment in animals fed diet with brassica sprouts (36).

The reason for the higher concentration of SCFAs in the stool during consumption of diet enriched with polyphenolic compounds may be associated with the specific monocarboxylate transporters (MCTs) for short fatty acids, which were reported in rat intestine. The primary role of MCTs is to mediate the absorption of SCFAs (i.e. acetate, propionate, and butyrate) from the intestinal lumen (38). These transporters may be inhibited by the natural amphiphilic compounds with divergent structures (e.g., quercetin and phloretin), and some stilbene derivatives presented also in food (39). However, in our investigation, no significant differences in concentration of all evaluated acids in various groups of healthy rats consuming brassica sprouts were observed, despite the fact that animals received the diet which is potentially very good source of polyphenols (25).

Our results suggest that thyroid damage indirectly affects the concentration of short-chain fatty acids in the stool. Moreover, a diet enriched with brassica sprouts, especially broccoli sprouts, influenced SCFAs concentration in stool samples of rats with thyroid damage. Further study is needed to evaluate the significance of the relationship between thyroid disorders and gastrointestinal tract function 
under conditions of increased amounts of broccoli sprouts in daily diet.

\section{Limitations}

We may expect more pronounced results if the experiment lasts longer, e.g. 12 or 16 weeks. The lack of qualitative analysis of gut microbiota is hindering the clear interpretation of results.

\section{Acknowledgment}

This research did not receive any specific grant from funding agencies in the public, commercial, or not-for-profit sectors. The authors declare that they have no conflict of interest.

\section{REFERENCES}

1. Ebert E.C.: J. Clin. Gastroenterol. 44, 402 (2010).

2. Daher R., Yazbeck T., Jaoude J.B., Abboud B.: World J. Gastroenterol. 15, 2834 (2009).

3. Yaylali O., Kirac S., Yilmaz M., Akin F., Yuksel D. et al.: Gastroenterol. Res. Pract. 7, (2007).

4. Maser C., Toset A., Roman S.: World J. Gastroenterol. 12, 3174 (2006).

5. Gullo L., Pezzilli R., Bellanova B., D’Ambrosi A., Alvisi V. et al.: Gastroenterology 100, 1392 (1991).

6. Jonderko G., Jonderko K., Marcisz C., Golab T.: Isr. J. Med. Sci. 33, 198 (1997).

7. Batke M., Cappell M.S.: Med. Clin. North Am. 92, 649 (2008).

8. Tenore A., Fasano A., Gasparini N., Sandomenico M.L., Ferrara A. et al.: J. Endocrinol. 151, 431 (1996).

9. Pucci E., Chiovato L., Pinchera A.: Int. J. Obesity 24, 109 (2000)

10. Biondi B.: Clin. Endocrinol. Metab. 95, 3614 (2010).

11. Ríos-Covián D., Ruas-Madiedo P., Margolles A., Gueimonde M., de los Reyes-Gavilán C.G. et al.: Front. Microbiol. 7, 1 (2016).

12. Roy C.C., Kien C.L., Bouthillier L., Levy E.: Nutr. Clin. Pract. 21, 351 (2006).

13. Lambert D.W., Wood I.S., Ellis A., ShiraziBeechey S.P.: Br. J. Cancer 86, 1262 (2002).

14. Jung T.H., Park J.H., Jeon W.M., Han K.S.: Nutr. Res. Pract. 9, 343 (2015).

15. Hamer H.M., Jonkers D., Venema K., Vanhoutvin S., Troost F.J. et al.: Aliment. Pharmacol. Ther. 27, 104 (2008).
16. Murphy K.G., Bloom S.R.: Nature 444, 854 (2006)

17. Frost G., Sleeth M.L., Sahuri-Arisoylu M., Lizarbe B., Cerdan S. et al.: Nat. Commun. 5, 3611 (2014).

18. Tolhurst G., Heffron H., Lam Y.S., Parker H.E., Habib A.M. et al.: Diabete. 61, 364 (2012).

19. Layden B.T., Angueira A.R., Brodsky M., Durai V., Lowe W.L. Jr.: Transl. Res. 161, 131 (2013).

20. Bjursell M., Admyre T., Göransson M., Marley A.E., Smith D.M. et al.: Am. J. Physiol. 300, 211 (2010).

21. Gao Z., Yin J., Zhang J., Ward R.E., Martin R.J. et al.: Diabetes. 58, 1509 (2009).

22. Kunc M., Gabrych A., Witkowski J.M.: Acta. Biochim. Pol. 63, 189 (2016).

23. Son H.Y., Nishikawa A., Ikeda T., Imazawa T., Kimura S. et al.: Jpn. J. Cancer Res. 92, 103 (2001).

24. Ikeda T., Nishikawa A., Son H.Y., Nakamura H., Miyauchi M. et al.: Jpn. J. Cancer Res. 92, 390 (2001).

25. Paśko P., Krośniak M., Prochownik E., TyszkaCzochara M., Fołta M. et al.: Biomed. Pharmacother. 97, 82 (2018).

26. Paśko P., Tyszka-Czochara M., Galanty A., Gdula-Argasińska J., Żmudzki P. et al.: ýPlant Foods Hum. Nutr. 73, 95 (2018).

27. Dobrowolska-Iwanek J., Zagrodzki P., Woźniakiewicz M., Woźniakiewicz A., ZwolińskaWcisło M. et al.: J. Pharm. Biomed. Anal. 30, 337 (2016).

28. http://vassarstats.net/rdiff.html (accessed 29 May 2018).

29. Lauritano E.C., Bilotta A.L., Gabrielli M., Scarpellini E., Lupascu A. et al.: J. Clin. Endocrinol. Metab. 92, 4180 (2007).

30. Oufir L.E., Barry J.L., Flourié B., Cherbut C., Cloarec D. et al.: Eur. J. Clin. Nutr. 54, 603 (2007)

31. Flint H.J., Duncan S.H., Scott K.P., Louis P.: Proc. Nutr. Soc. 74, 13 (2015).

32. Lerner A., Jeremias P., Matthias T.: Endocr. Connect. 6, R52 (2017).

33. DiStefano J.J., de Luze A., Nguyen T.T.: Am. J. Physiol. 264, E966 (1993).

34. Fekete C., Gereben B., Doleschall M., Harney J.W., Dora J.M. et al.: Endocrinology 145, 1649 (2004).

35. Bazzocco S., Mattila I., Guyot S., Renard C.M., Aura A.M.: Eur. J. Nutr. 47, 442 (2008).

36. Boto-Ordóńez M., Urpi-Sarda M., QueipoOrtuńo M.I., Tulipani S., Tinahones F.J. et al.: Food Funct. 5, 1932 (2014). 
37. Kaczmarek J.L., Charron C.S., Novotny J.A., Jeffery E.H., Seifried H.E. et al.: FASEB J. 31, Supplement 965.18. (2017).

38. Adijanto J., Philp N.J.: Curr. Top. Membr. 70, 275 (2012).
39. Wolf K.K., Paine M.F., Watkins P.B.: Metabolic barrier of the gastrointestinal tract, in McQueen ChA (ed) Comprehensive Toxicology. pp. 54-71, Elsevier, United Kingdom 2010.

Received: 28.06 .2019 